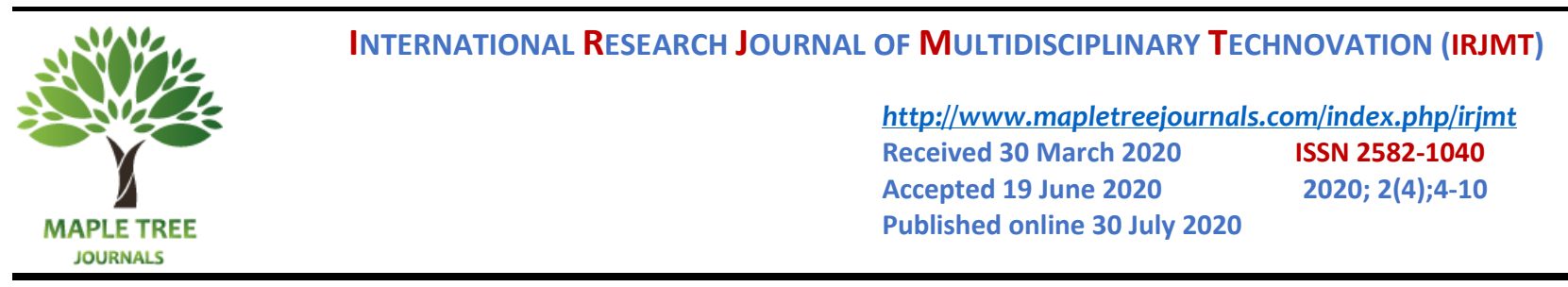

\title{
Social Communicative Extraction Analysis
}

\author{
Santhosh Kumar $S^{1,}$ *, Vishnu Vardhan $S^{1}$, Wasim Jaffar M ${ }^{1}$, Sultan Saleem A ${ }^{2}$, \\ Sharmasth Vali $\mathbf{Y}^{2}$ \\ ${ }^{1}$ Department of Computer Science and Engineering, Dhanalakshmi College of Engineering, Chennai - 601 301, \\ Tamil Nadu, India. \\ *Corresponding author E-Mail ID: santhoshdhanss@gmail.com
}

DOI: https://doi.org/10.34256/irjmt2042

\begin{abstract}
The distinguishing proof of online networking networks has as of late been of significant worry, since clients taking an interest in such networks can add to viral showcasing efforts. Right now center around clients' correspondence considering character as a key trademark for recognizing informative systems for example systems with high data streams. We portray the Twitter Personality based Communicative Communities Extraction (T-PCCE) framework that recognizes the most informative networks in a Twitter organize chart thinking about clients' character. We at that point grow existing methodologies as a part of client's character extraction by collecting information that speak to a few parts of client conduct utilizing AI strategies. We utilize a current measured quality based network discovery calculation and we expand it by embeddings a post-preparing step that dispenses with diagram edges dependent on clients' character. The adequacy of our methodology is exhibited by testing the Twitter diagram and looking at the correspondence quality of the removed networks with and without considering the character factor. We characterize a few measurements to tally the quality of correspondence inside every network. Our algorithmic system and the resulting usage utilize the cloud foundation and utilize the MapReduce Programming Environment. Our outcomes show that the T-PCCE framework makes the most informative networks.
\end{abstract}

Keywords: T-PCCE, Data Streams, Analysis, AI, Twitter.

\section{Introduction}

As of late with the develop of web-based social networking the job of system as a stage for data dispersion has pulled in the consideration of organizations. This paper is investigating the issue of distinguishing networks of basic enthusiasm, looking at clients' correspondence conduct and character as a shared opinion for network development. The focal point of this work is on the correspondence part of networks and subsequently is separated from existing and progressively broad work on the distinguishing proof of persuasive clients which is for the most part dependent on clients static profile and system associations. Our perspective on making open networks, is to utilize the "follow" connections of Twitter and concentrate the symboliclinks that rise through Twitter cooperations and clients characters, distinguishing networks of high data stream. Such people group can be the point of focused advertising exercises since the data will stream quick and simple.

Right now think about, that a main quality of computerized informal organizations is their Big Data nature. As Boyd [4] referenced, Big Data is about individuals and their cooperations 
henceforth we utilize the plenitude of data on clients' message exchanges through tweets among their friends to extricate clients' character data to deduce arrange social conduct.

\subsection{Existing System}

Newman proposed a calculation that utilizes particularity as the proportion of segment quality and pick the segment that would expand measured quality. The measured quality based model has been end up being an important route for distinguishing the nearness of network structures in systems since it evaluates the nature of the partitioned networks. Thick inward associations inside networks and hardly any associations between them is the paradigm for choosing networks segment. This model is in understanding to the thinking of the Twitter arrange where gatherings of clients with solid connections are interrelated through the "follow" associations. Right now are keen on recognizing a built up network location calculation and expand it with clients' character data so as to distinguish networks that are progressively open. Among the current methodologies for network recognition, the boost of the system measured quality methodology is notable and generally received. Since the issue of augmenting the measured quality of the system in NP-complete, the most mainstream strategy for seclusion based network location has been presented in the Louvain technique.

\subsection{Literature Survey}

\section{Source Personality and Persuasiveness: Big Five Predispositions to Being Persuasive and the Role of Message Involvement}

In the present investigations we consolidate a Person Situation viewpoint into the investigation of the influence source. In particular, we intended to distinguish the character qualities of the convincing individual and test the directing job of target and source inclusion. Demonstrated that source Extraversion and Openness to Experience were decidedly, and Neuroticism contrarily, related with source enticement. In Study 2 ( $\mathrm{N}=148$, Mage $=24.3,61 \%$ female), we controlled the degree of association and for the most part repeated the outcomes from Study 1, at the same time, comparing with our expectations, just when inclusion was low. Our discoveries show the importance of an interactionist way to deal with the investigation of influence, featuring the job of character in the investigation of the influence source.

\section{Inferring Topic-Dependent Influence Roles of Twitter Users}

Twitter, as one of the most famous internet based life stages, gives a helpful method to individuals to convey and communicate with one another. It has been very much perceived that impact exists during clients' communications. Some pioneer contemplates on finding persuasive clients have been accounted for in the writing, however they don't recognize distinctive impact jobs, which are of incredible incentive for different showcasing purposes. Right now, push a stage ahead attempting to additionally recognize impact jobs of Twitter clients in a specific theme. By characterizing three perspectives on highlights identifying with subject, slant and prominence separately, we propose a Multi-see Influence Role Clustering (MIRC) calculation to gather Twitter clients into five classifications. Test results show the adequacy of the proposed approach in construing impact jobs.

\section{Proposed System}

Our commitments are in a few perspectives: right off the bat, we expand the current methodologies for character based clients' conduct extraction from internet based life information by expanding the dimensionality of client's information object at that point we distinguish the mining calculations that best fit every character characteristic and broaden network recognition calculations by including a post processing step that represents clients' character. Moreover, a 
bound together structure that joins character mining and network location to address the issue of distinguishing informative networks is proposed. We build up an applied philosophy for integrating sensible datasets dependent on genuine information and alter the network location calculation to the setting of Hadoop using a few bunch hubs, consequently our proposed strategy can without much of a stretch scale up.

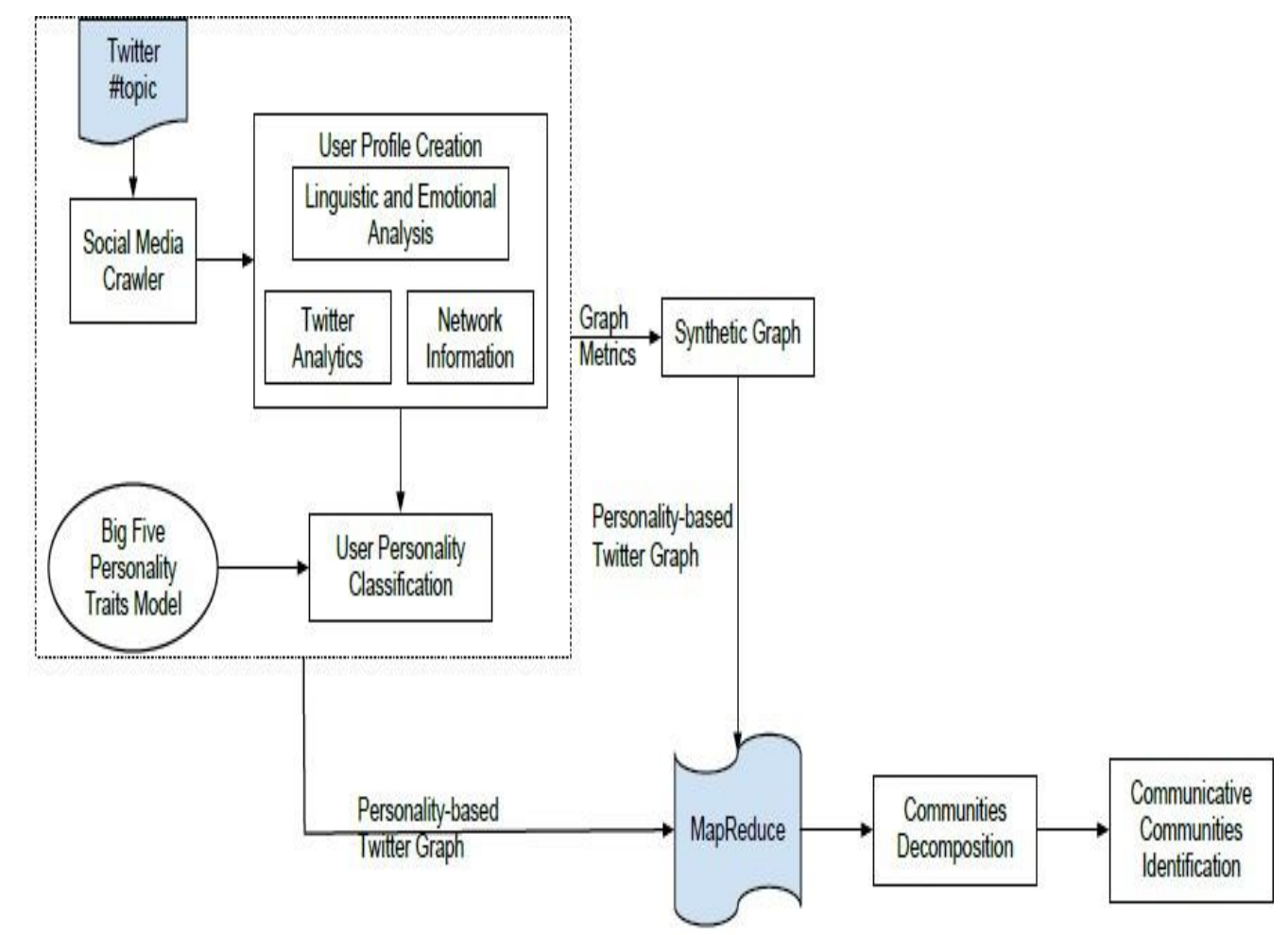

Fig 1. Proposed System Architecture

\subsection{Materials and Methods}

Structural Features: Through a Twitter application, we can gather data about the client's egocentric system. We originally acquired a rundown of companions. We were keen on thickness, and Twitter gives some data about connections between a client's companions. A different question must be made for each pair of clients to decide whether they are or are not companions. It was impractical to present an inquiry for each pair of companions in light of the fact that the Twitter application would break Twitter restricts the time an application can run, and since each question is sent over the system, execution turns into an issue. Along these lines, we examined 2,000 one of a kind sets of companions from a client's egocentric system and utilized that to decide the thickness of the system, for example what level of potential edges between companions exist (see fig 2).

Personal Information: Clients give an abundance of individual data. We gathered everything accessible, despite the fact that a few highlights would end up having no utilization in our examination. The crude information included highlights like the client's name, birthday, relationship status, religion, training history, sexual orientation, and old neighborhood. The greater part of this data was not required, so a few clients did exclude all data. Where conceivable, we made extra highlights that demonstrated whether the client had incorporated the data (for 
example was a religion or old neighborhood gave or not), or what number of things were recorded (for example what number of instructive encounters were recorded). These additional highlights ended up being substantially more helpful and prescient than the first crude information. For instance, from 279 clients, 111 recorded a religion. Inside those 111 individuals were 82 unique sections. This makes a space too scanty to even consider doing any factual investigation, however simply knowing whether an individual recorded a religion or not uncovers bits of knowledge into what they are eager to share (see fig 3 ).

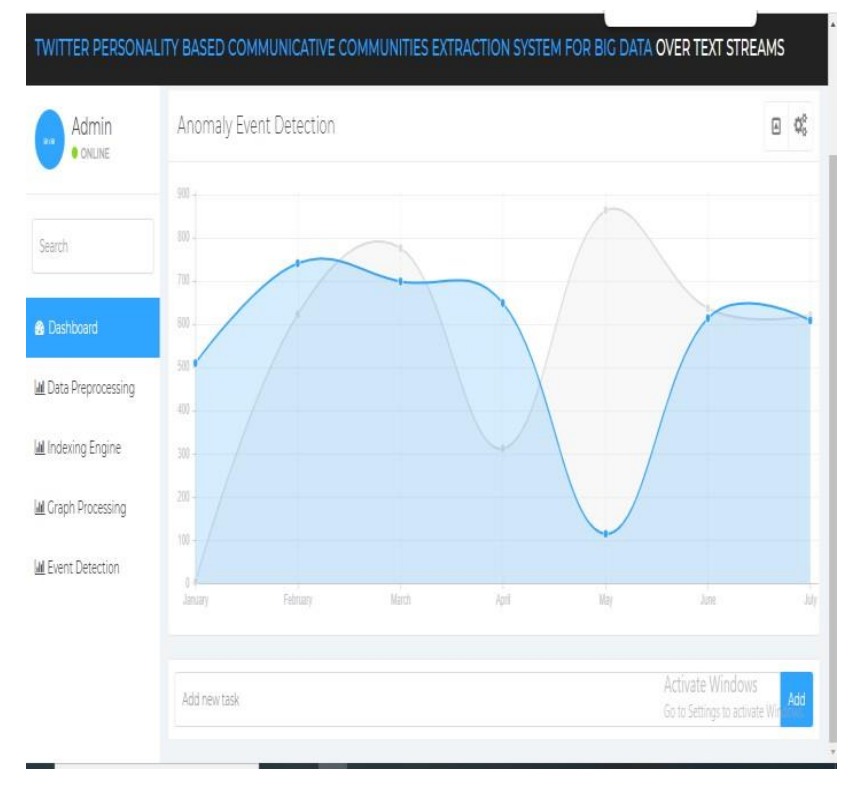

Fig 2. Structural Features

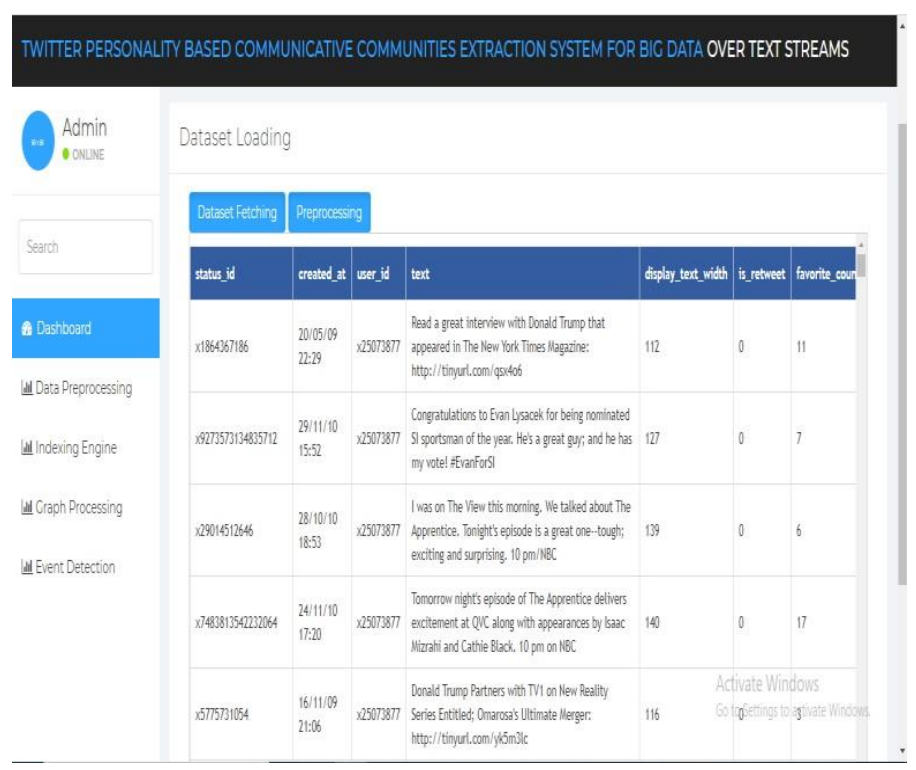

Fig 3. Personal Information

Activities and Preferences: Giving arrangements of individual exercises or most loved things has consistently been a piece of Twitter. Clients list most loved TV appears, films, music, book, cites, just as political and authoritative affiliations and most loved exercises. Similar to the case with religion portrayed over, the space is far to scanty to do any examination over the genuine sections in these fields, so we made more buddy measures. For arrangements of most loved things and exercises, we included the quantity of characters in the passage, generally estimating how a lot of 
data the client gave in each field. This included estimations of 0 for clients who didn't supply any data. For authoritative affiliations, we checked the number recorded and for political affiliations, we basically estimated whether it was shared or not (see fig 4).

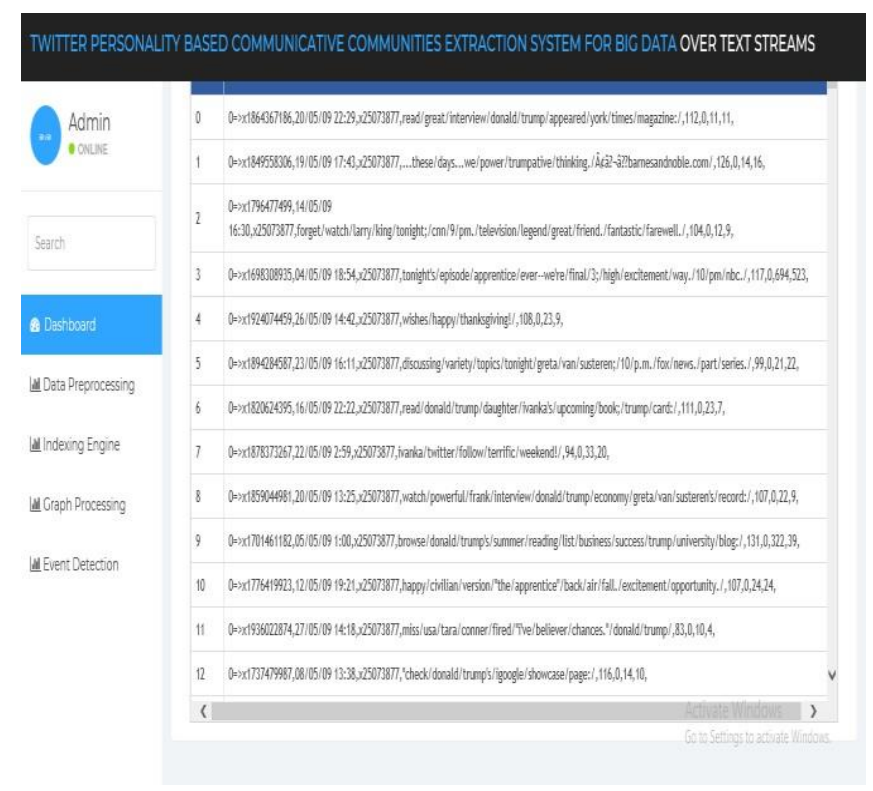

Fig 4. Activities and Preference

Language Features: Like the exercises and likes portrayed above, clients additionally have chances to share increasingly close to home composed data through the "About Me" and "ad spot" message in their profiles, and through announcements. We gathered these passages and furthermore added highlights to quantify the character length of every section.

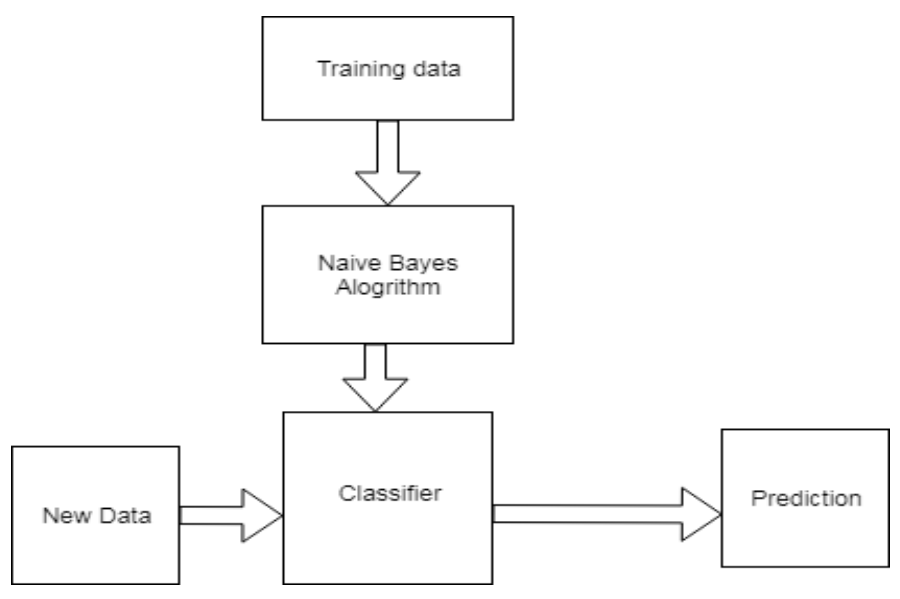

Fig 5. Block Diagram

\section{System Design}

System has 3 units Input unit, processing unit, output unit (see fig 5).

A) Input Unit:

The User is act as an input unit, which we can select the profile of the use according to the tweet they written about the particular incident.

B) Processing Unit: 
The processing unit where we can collect the personal information about the users. Users provide a wealth of personal information. We collected everything available, even though some features would turn out to have no use in our analysis.

\section{C) Output Unit:}

Our output in this work is that personalityis influencing communities communication hence we can have smaller communities with the same number of Tweets and thus identify dense communication communities.

\section{Experiment and Results}

Our goal is to recognize networks that are however much informative as could reasonably be expected while simultaneously having less hubs. Such people group can give message correspondence among the most individuals rather than bigger networks where correspondence isn't frequently; we can describe these networks as "thick" in data trade. Our outcomes show that networks made after our methodology are more open than the Louvain strategy in all cases (see fig 6 and fig 7).

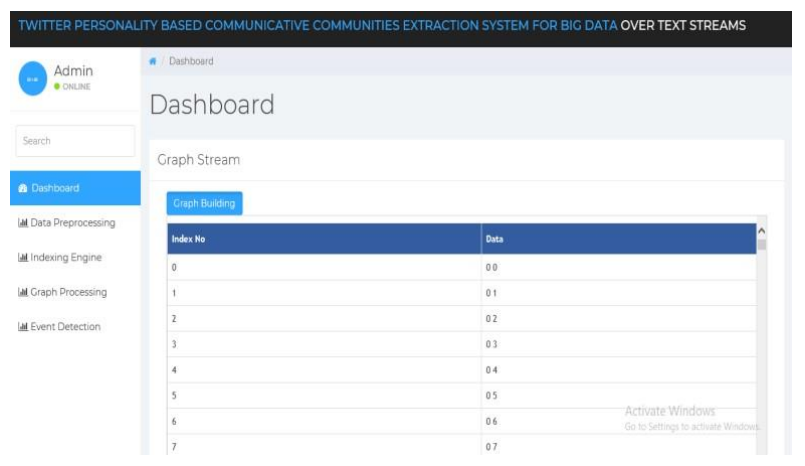

Fig 6. Graph Streams

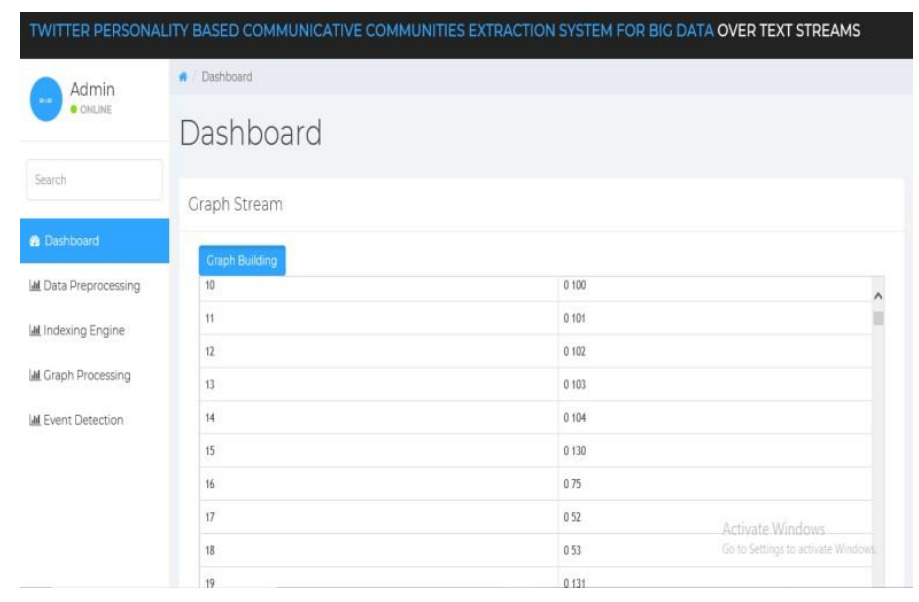

Fig 7. Graph Streams

\section{Conclusion}

The open networks are made dependent on a few varieties of measured quality based network discovery, where character is likewise considered in a post-preparing step. At long last, the examination of the proposed varieties and the underlying network recognition calculation is assessed dependent on measurements that check the action level of the best three networks. Our work is the utilization of the MapReduce programming condition, utilizing Hadoop framework; 
right now, approach can be viewed as versatile and ready to deal with datasets of enormous size. Besides, we proposed a technique for making manufactured datasets dependent on genuine ones.

\section{References}

[1] F. Alam, E. A. Stepanov, and G. Riccardi. Character attributes acknowledgment on informal organization - facebook. Computational Personality Recognition, 2013.

[2] D. Boyd. Security and exposure with regards to large information. In Keynote Talk of the WWW, 2010.

[3] F. Celli and L. Rossi. The job of enthusiastic dependability in twitter discussions. In Semantic Analysis in Social Media, pages 10-17, 2012.

[4] C. Chen, D. Gao, W. Li, and Y. Hou. Deducing subject ward impact jobs of twitter clients. In SIGIR, pages 1203-1206, 2014.

[5] P. Chopade, J. Zhan, and M. Bikdash. Hub qualities and edge structure for huge scope large information arrange examination and network identification. In International Symposium on Technologies for Homeland Security (HST), (2015) 1-8.

[6] D. Cosley, D. P. Huttenlocher, J. M. Kleinberg, X. Lan, and S. Suri. Successive impact models in interpersonal organizations. In International Conference on Weblogs and Social Media (ICWSM), 2010.

[7] P. Cui, F. Wang, S. Liu, M. Ou, S. Yang, and L. Sun. Who should share what? thing level social impact forecast for clients and posts positioning. In SIGIR, (2011)185-194.

[8] P. Cui, Z. Wang, and Z. Su. What recordings are comparative with you?: Learning a typical credited portrayal for video suggestion. In ACM International Conference on Multimedia (MM), (2014) 597-606.

[9] P. Cui, W. Zhu, T. Chua, and R. Jain. Social-detected interactive media registering. IEEE Multi Media, 23 (2016) 92-96.

[10] M. D urr, V. Protschky, and C. Linnhoff-Popien. Displaying informal organization cooperation diagrams. In ASONAM, (2012) 660-667.

\section{Acknowledgement Nil}

Funding

This study was not funded by any grant

\section{Conflict of interest}

None of the authors have any conflicts of interest to declare.

\section{About The License}

The text of this article is licensed under a Creative Commons Attribution 4.0 International License

\section{Cite this Article}

Santhosh Kumar S, Vishnu Vardhan S, Wasim Jaffar M, Sultan Saleem A, Sharmasth Vali Y, Social Communicative Extraction Analysis, International Research Journal of Multidisciplinary Technovation, Vol 2, Iss 4 (2020) 4-10.

DOI: https://doi.org/10.34256/irimt2042 